\title{
Supplementary Materials: Using informative priors in the estimation of mixtures over time with application to aerosol particle size distributions
}

\author{
September 24, 2013
}

\section{Simulated dataset (D2): Noisy data}

Figure 1 shows the results of analysis using dataset D2, using the informed prior approach with $\theta=0.5$ and penalised prior approach with $\phi=0.08$ and the independent prior approach. The values for $\theta$ and $\phi$ were chosen to allow prior information to be influential, but not overwhelm the posterior estimates. Interestingly it is evident that smoothing on the weights results in compensatory measures by both $\mu$ and $\sigma$. The compensatory measures appear to be more pronounced when $\lambda$ is volatile over time. In this case, the prior is imposing larger adjustments away from the data at each time point. We see this most clearly in the results for the first two components, but not for the third component. A possible explanation for this observation is that for the first component, $\mu$ is able to adjust to a higher value which is supportive of a greater value for $\lambda$, and in some sense borrow support from the second component. However, for the third component, $\mu$ is not able to increase or decrease in support of a lower value for $\lambda$ by borrowing support from a nearby component.

In general, from the results of smoothing on $\mu, \sigma$ and $\lambda$ for the informed prior and penalised prior approaches it appears that large adjustments to one parameter (e.g. from volatility in some time periods) are not supported unless compensatory measures can be taken by the other parameters. From exploratory analysis these compensatory measures appear to be independent of sample size.

Figure 2 provides the results of the hierarchical model for $\mu$ (green and blue) and the actual data (black), with $\sigma$ and $\lambda$ estimated using the independent approach. For these results, $\epsilon_{\mu}^{(d)}$; $\epsilon_{\mu}^{(d)}$ was fixed at 0.36 using the information from the independent approach; and $\epsilon_{\phi}^{(s)}$ was set at 0.04 , a smaller multiple of $\epsilon_{\mu}^{(d)}$ as discussed previously. From Figure 2, we can see that the estimates for $\phi$ are a smooth version of the much noisier estimates for $\mu$. 

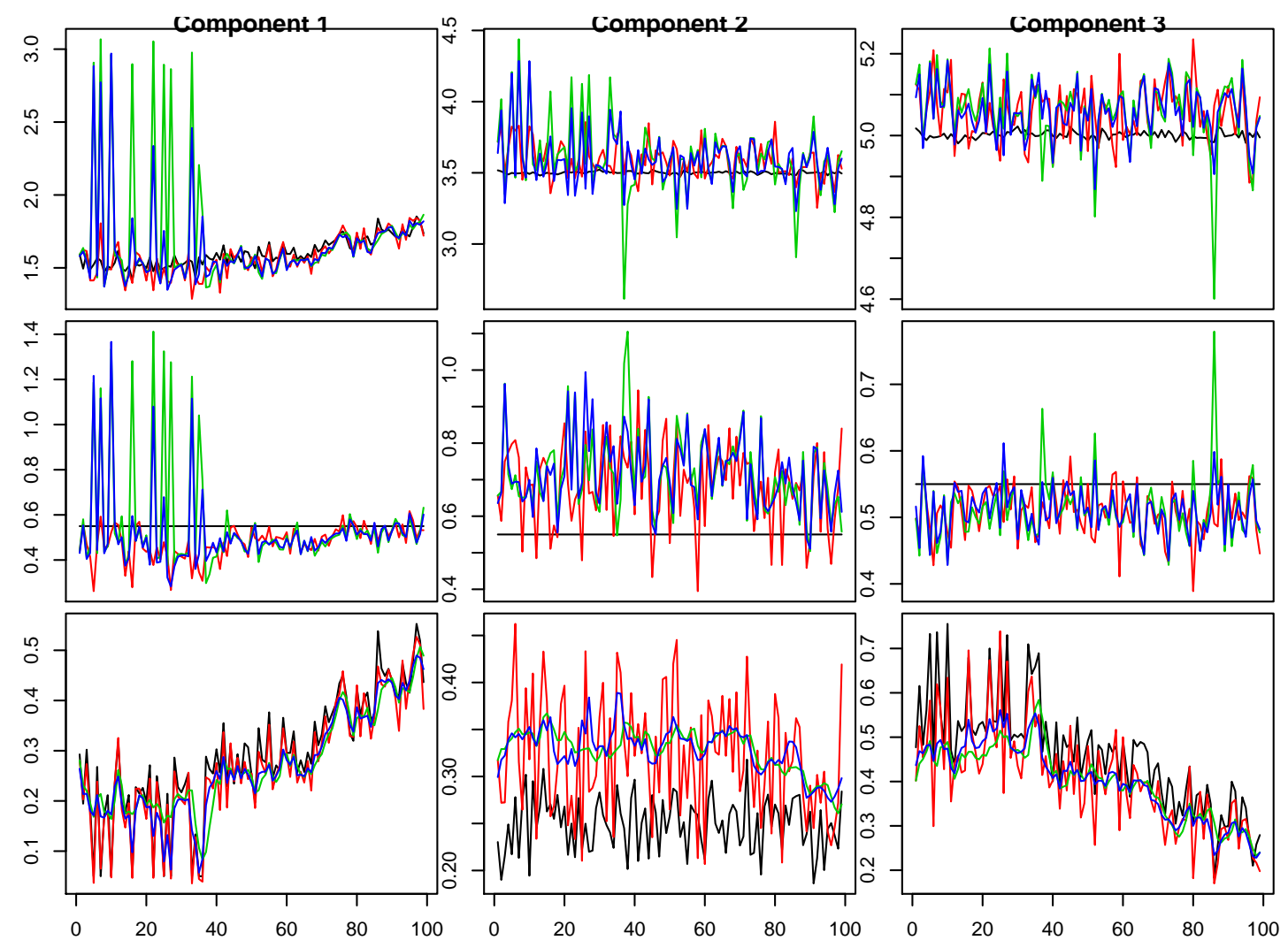

Figure 1: Results for simulated dataset D2 (Noisy data) with growth and influx of new particles for Component 1. Plot of estimated posterior mean for parameters ( $\mu$ (top), $\sigma^{2}$ (middle) and $\lambda$ (bottom)) over time (x-axis) for different approaches: Simulated data (Black); Independent (Red); Informed Prior (Green); Penalised Prior (Blue). The columns represent the components (Components 1 to 3 ). 

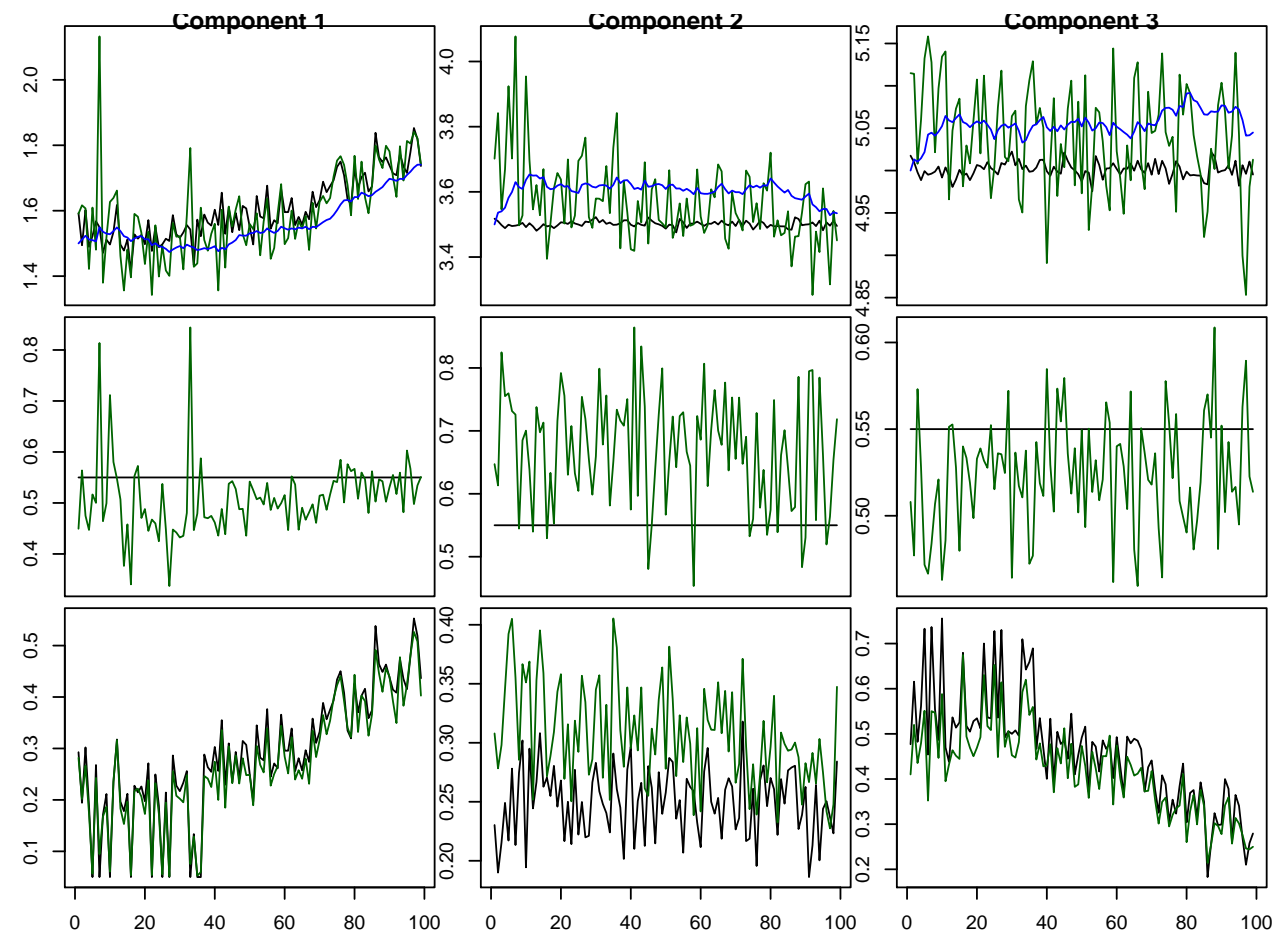

Figure 2: Results for simulated dataset D2 (Noisy data) with growth and influx of new particles for Component 1. Plot of estimated posterior mean for parameters ( $\mu$ (top), $\sigma^{2}$ (middle), $\lambda$ (bottom)) over time (x-axis) for Hierarchical approach: Actual data (Black line); Hierarchical approach for $\mu$ (Dark Green line); $\phi$ (Blue line). The columns represent the components (Components 1 to 3). 
Figure 3 provides the results of the hierarchical model for $\lambda$ (green and blue) and the actual data (black), with $\mu$ and $\sigma$ estimated using the independent approach. For these results, $\epsilon_{\lambda}^{(d)}$ and $\epsilon_{\gamma}^{(s)}$ are fixed at 0.2 and 0.015 , respectively, based upon the variability of $\lambda$ from the results of the independent approach. From Figure 3 the estimates for $\gamma$ roughly follow the more variable estimates for $\lambda$ over time. In contrast to the results from the informed prior (Figure 1), we do not see any large compensatory adjustments being made to parameters by using a hierarchically based informative prior for $\gamma$. Apart from estimates for $\gamma$, the variability of the other parameter estimates are comparable to the results from the independent approach.
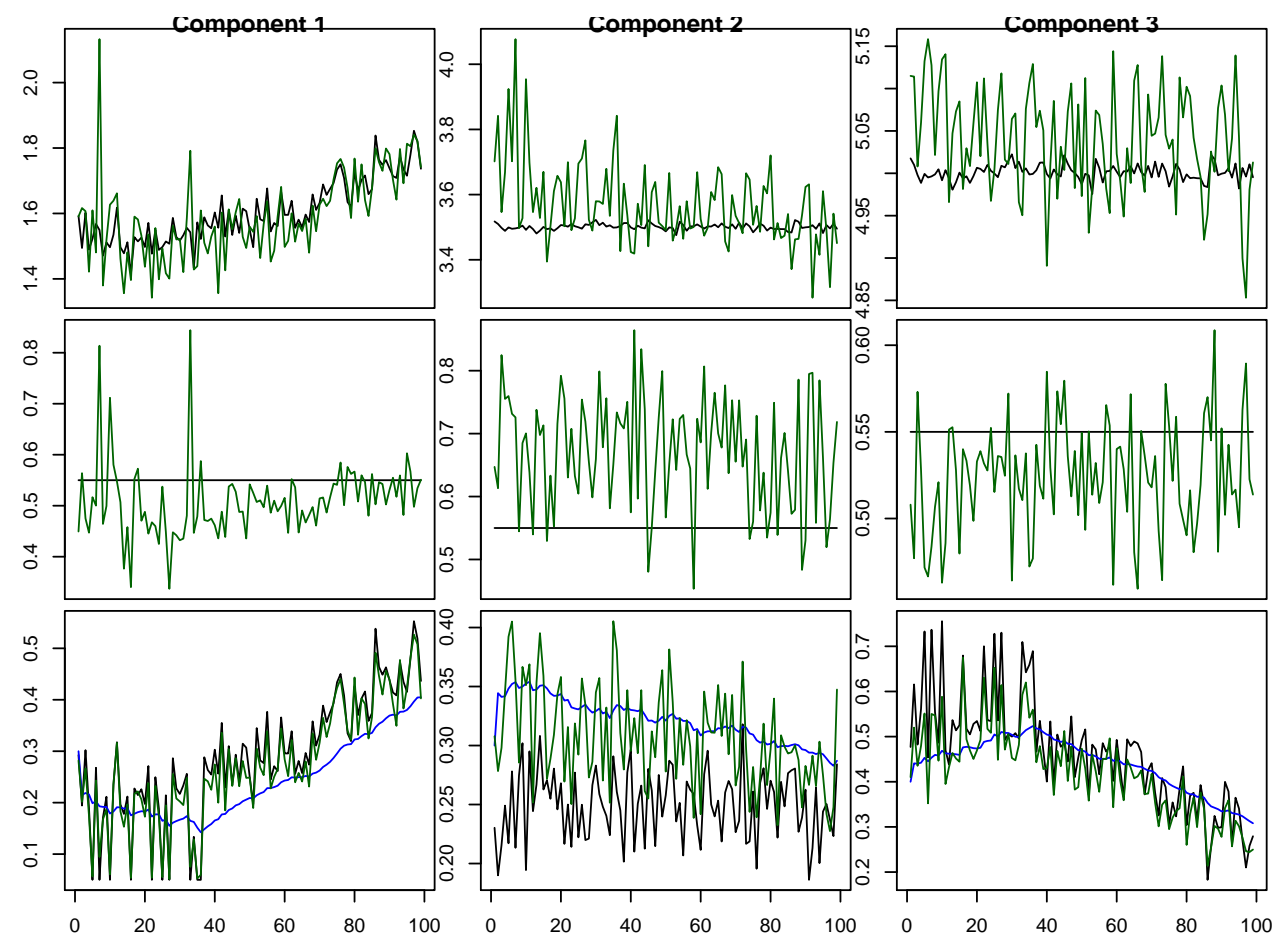

Figure 3: Results for simulated dataset D2 (Noisy data) with growth and influx of new particles for Component 1. Plot of estimated posterior mean for parameters ( $\mu$ (top), $\sigma^{2}$ (middle), $\lambda$ (bottom)) over time (x-axis) for Hierarchical approach: Actual data (Black line); Hierarchical approach for $\lambda$ (Dark Green line); $\gamma$ (Blue line). The columns represent the components (Components 1 to 3).

Setting $\epsilon_{\lambda}^{(d)}$ equal to the same value as $\epsilon_{\gamma}^{(s)}(=0.015$, relatively little allowance for noise) we see similar results to using the informative prior approach for $\lambda$ (Figure 1) discussed previously, where large compensatory adjustments are made by $\mu$ and $\sigma$ in response. At the other end, if we set $\epsilon_{\gamma}^{(s)}$ equal to $\epsilon_{\lambda}^{(d)}(=0.2)$ we see results similar to using the independent approach (see Figure 1).

In analyses not shown here, we compared the results from using a three period centred moving average on the weight for the informed prior to the results of using the penalised prior to assess 
whether the form specified by the penalised prior had a different influence on the results. We found the results from using both approaches to be largely the same. 